\title{
Alternative kinetic energy metrics for Lagrangian systems
}

\author{
W. Sarlet ${ }^{a, b}$ and G. Prince ${ }^{b}$ \\ ${ }^{a}$ Department of Mathematics, Ghent University \\ Krijgslaan 281, B-9000 Ghent, Belgium \\ ${ }^{b}$ Department of Mathematics and Statistics, La Trobe University \\ Melbourne, Victoria 3086, Australia
}

\begin{abstract}
We examine Lagrangian systems on $\mathbb{R}^{n}$ with standard kinetic energy terms for the possibility of additional, alternative Lagrangians with kinetic energy metrics different to the Euclidean one. Using the techniques of the inverse problem in the calculus of variations we find necessary and sufficient conditions for the existence of such Lagrangians. We illustrate the problem in dimensions two and three with quadratic and cubic potentials. As an aside we show that the well-known anomalous Lagrangians for the Coulomb problem can be removed by switching on a magnetic field, providing an appealing resolution of the ambiguous quantisations of the hydrogen atom.
\end{abstract}

\section{Introduction}

The inverse problem of Lagrangian mechanics is about deciding whether a given system of second-order ordinary differential equations (SODE for short) can be recast into the form of Euler-Lagrange equations for some Lagrangian $L$. The literature about this subject is vast and so are the different techniques and mathematical tools which have been used in its development. For a sample of such different approaches (listed in chronological order) we refer to $[13,12,2,6,1,3]$; many more references can be found for example in the review paper [9] and it is perhaps worth mentioning that a generalization of the problem, using the geometric calculus developed in [10], has recently been presented in [11].

Many of the contributions referred to above make use of different, but quite advanced differential geometric methods, and often aim at obtaining classification type results concerning SODEs which admit a Lagrangian representation. We wish to emphasise that our purpose in the present paper is to analyse a very concrete subclass of systems, for which there exist multiple Lagrangian formulations, and which further have an obvious physical connotation. Accordingly, we shall keep reference to the differential geometric background to a minimum and express all conditions of interest in analytical terms. 
The SODEs we start from are autonomous differential equations on in normal form, say

$$
\ddot{x}^{a}=F^{a}(x, \dot{x}), \quad a=1, \ldots, n,
$$

which geometrically come from a second-order equation (vector) field, called $\Gamma$, on the tangent bundle $T M$ of some $n$ dimensional configuration manifold $M$. Coordinates on $T M$ will be denoted by $\left(x^{a}, \dot{x}^{a}\right)$, so

$$
\Gamma=\dot{x}^{a} \frac{\partial}{\partial x^{a}}+F^{a}(x, \dot{x}) \frac{\partial}{\partial \dot{x}^{a}} .
$$

In the autonomous setting, the inverse problem we are addressing is the existence and uniqueness question for a multiplier matrix $g_{a b}(x, \dot{x})$, such that

$$
g_{a b}\left(\ddot{x}^{b}-F^{b}\right) \equiv \frac{d}{d t}\left(\frac{\partial L}{\partial \dot{x}^{a}}\right)-\frac{\partial L}{\partial x^{a}},
$$

for some Lagrangian function $L(x, \dot{x})$. Necessary and sufficient conditions for the existence of a Lagrangian are generally referred to as the Helmholtz conditions, and when regarded as conditions that a non-singular multiplier must satisfy, they read as follows:

$$
g_{a b}=g_{b a}, \quad \Gamma\left(g_{a b}\right)=g_{a c} \Gamma_{b}^{c}+g_{b c} \Gamma_{a}^{c}, \quad g_{a c} \Phi_{b}^{c}=g_{b c} \Phi_{a}^{c}, \quad \frac{\partial g_{a b}}{\partial \dot{x}^{c}}=\frac{\partial g_{a c}}{\partial \dot{x}^{b}},
$$

where

$$
\Gamma_{b}^{a}:=-\frac{1}{2} \frac{\partial F^{a}}{\partial \dot{x}^{b}}, \quad \Phi_{b}^{a}:=-\frac{\partial F^{a}}{\partial x^{b}}-\Gamma_{b}^{c} \Gamma_{c}^{a}-\Gamma\left(\Gamma_{b}^{a}\right) .
$$

The interested reader can look up the direct, coordinate free formulation of the conditions (3), which was developed in [10], in the introduction of [1], for example. We should at least say that the functions $\Gamma_{b}^{a}$ are the connection coefficients of the canonical nonlinear connection which comes with the given SODE $\Gamma$ and that they in turn give rise to the important concept of dynamical covariant derivative, a derivation $\nabla$, of degree 0 , determined by

$$
\nabla \frac{\partial}{\partial x^{b}}=\Gamma_{b}^{a} \frac{\partial}{\partial x^{a}}, \quad \nabla d x^{a}=-\Gamma_{b}^{a} d x^{b}, \quad \nabla F=\Gamma(F), F \in C^{\infty}(T M) .
$$

Importantly, an integrability study of the partial differential equations for the $g_{a b}$ leads to further algebraic restrictions, which may give interesting extra information (see e.g. [13]). For future reference, we mention one of them, called the curvature condition. It reads

$$
g_{a r} R_{b c}^{r}+g_{b r} R_{c a}^{r}+g_{c r} R_{a b}^{r}=0 .
$$

Here $R_{b c}^{a}$ are the components of the curvature tensor of the non-linear connection. Their coordinate expression is

$$
R_{b c}^{a}:=H_{c}\left(\Gamma_{b}^{a}\right)-H_{b}\left(\Gamma_{c}^{a}\right), \quad \text { where } \quad H_{c}:=\frac{\partial}{\partial x^{c}}-\Gamma_{c}^{r} \frac{\partial}{\partial \dot{x}^{r}} .
$$


Of course, if we can find a solution of the equations (3), we don't have to worry about such further conditions, since they are merely integrability conditions for the existence of solutions.

The restricted, concrete question we shall address in this paper is the characterization of SODEs on $\mathbb{R}^{n}$ which have a Lagrangian representation with a Euclidean kinetic energy term, and which admit alternative Lagrangian representations including those for which the multiplier matrix does not depend on the velocities. The main conditions about detecting such systems are developed in section 2. In section 3, it is shown that such systems actually decouple into lower dimensional Lagrangian systems in coordinates which diagonalise the alternative multiplier. A number of explicit examples are worked out in section 4 . The final section concerns the Coulomb/Kepler problem on $\mathbb{R}^{3}$. This system is known to admit alternative Lagrangians which lead to anomalous quantisations for the hydrogen atom [7]. Although this is a case where the alternative multiplier depends on the velocities, it fits with the spirit and results of section 2 . We show that turning on a constant magnetic field removes all the Lagrangian degeneracy in this problem (the sole and unambiguous quantisation being the hydrogen atom with Zeeman effect).

\section{A particular class of Lagrangian systems}

Suppose $\Gamma$ is a soDE field on $T M$ with the property that, in some coordinate system, there exists a constant (non-singular) multiplier matrix for the inverse problem. Such a constant symmetric $g_{a b}$ is the Hessian of the function $T=\frac{1}{2} g_{a b} \dot{x}^{a} \dot{x}^{b}$ and the preceding statement means that there exists a Lagrangian $L$ for the given SODE, in the class of functions with the same Hessian. Since the freedom in choosing an $L$ with Hessian $g_{a b}$ amounts to adding to $T$ a linear function in the velocities, the Lagrangian which we assume to exist will be of the form

$$
L=T+A_{c}(x) \dot{x}^{c}-V(x) .
$$

The identity (2) produces

$$
F_{a}:=g_{a b} F^{b}=\left(\frac{\partial A_{b}}{\partial x^{a}}-\frac{\partial A_{a}}{\partial x^{b}}\right) \dot{x}^{b}-\frac{\partial V}{\partial x^{a}},
$$

so that the 'forces' $F_{a}$ are necessarily of the form of 'generalised electromagnetic forces' in the coordinates under consideration. We shall restrict ourselves here to the case where $M=\mathbb{R}^{n}$ with the Euclidean metric which gives rise to the given constant multiplier being the unit matrix in cartesian coordinates $\left(x^{a}\right): g_{a b}=\delta_{a b}$; the corresponding Lagrangian will be denoted $L_{0}$. The right-hand sides of the given equations will then be of the form (with $A^{a}=\delta^{a b} A_{b}$ )

$$
F^{a}=\left(\frac{\partial A^{b}}{\partial x^{a}}-\frac{\partial A^{a}}{\partial x^{b}}\right) \dot{x}^{b}-\frac{\partial V}{\partial x^{a}}
$$


for some functions $V$ and $A^{a}$ on $\mathbb{R}^{n}$. It follows that the connection coefficients are skew-symmetric:

$$
\Gamma_{b}^{a}=-\frac{1}{2} \frac{\partial F^{a}}{\partial \dot{x}^{b}}=\frac{1}{2}\left(\frac{\partial A^{a}}{\partial x^{b}}-\frac{\partial A^{b}}{\partial x^{a}}\right)
$$

while

$$
\Phi_{c}^{a}=\frac{1}{2}\left(\frac{\partial^{2} A^{a}}{\partial x^{c} \partial x^{b}}+\frac{\partial^{2} A^{c}}{\partial x^{a} \partial x^{b}}-2 \frac{\partial^{2} A^{b}}{\partial x^{a} \partial x^{c}}\right) \dot{x}^{b}-\Gamma_{b}^{a} \Gamma_{c}^{b}+\frac{\partial^{2} V}{\partial x^{a} \partial x^{c}}
$$

clearly is symmetric.

We want to investigate the possibility of a second multiplier $g$ for such systems which does not depend on the velocities. If $g$ does not depend on the velocities, it follows from the condition

$$
\Gamma\left(g_{a b}\right)=\frac{\partial g_{a b}}{\partial x^{c}} \dot{x}^{c}=g_{a c} \Gamma_{b}^{c}+g_{b c} \Gamma_{a}^{c},
$$

that $g$ must actually be constant and the matrix $\left(g_{a c} \Gamma_{b}^{c}\right)$ must be skew-symmetric which, in view of the skew-symmetry of the $\Gamma_{b}^{a}$, is equivalent to saying that $g$ and $\left(\Gamma_{b}^{a}\right)$ commute. The remaining Helmholtz condition reads $g_{a c} \Phi_{b}^{c}=g_{b c} \Phi_{a}^{c}$ and is, because of the symmetry of $\Phi$, also equivalent to the commutativity of $g$ and $\Phi$. From the definition of $\Phi$ and the structure of the functions $F^{a}$, it is straightforward to show that this condition will be satisfied if and only if

$$
g_{a c} \frac{\partial^{2} V}{\partial x^{c} \partial x^{b}}=g_{b c} \frac{\partial^{2} V}{\partial x^{c} \partial x^{a}},
$$

and

$$
g_{a c} \frac{\partial \Gamma_{r}^{c}}{\partial x^{b}}-g_{b c} \frac{\partial \Gamma_{r}^{c}}{\partial x^{a}}=g_{a c} \frac{\partial \Gamma_{b}^{c}}{\partial x^{r}} .
$$

The first of these puts rather severe restrictions on admissible potentials. Putting $\gamma_{a b}:=$ $g_{a c} \Gamma_{b}^{c}$, the second condition can be written as

$$
\frac{\partial \gamma_{a r}}{\partial x^{b}}+\frac{\partial \gamma_{r b}}{\partial x^{a}}+\frac{\partial \gamma_{b a}}{\partial x^{r}}=0 .
$$

Since $\gamma_{a b}$ is skew-symmetric, this expresses the closure of a 2 -form, so that there will exist local functions $B_{a}$ with

$$
\gamma_{a b}=g_{a c} \Gamma_{b}^{c}=\frac{\partial B_{a}}{\partial x^{b}}-\frac{\partial B_{b}}{\partial x^{a}} .
$$

One can further show that the cyclic sum condition on the $\gamma_{a b}$ is the curvature condition to be satisfied by the multiplier.

Summarizing, we have:

Proposition 1. Assume that the SODE $\Gamma$ on $\mathbb{R}^{n}$ admits a coordinate representation for which $\delta_{a b}$ is a multiplier matrix for the inverse problem of Lagrangian mechanics. Then the right-hand sides of the equations (1) are of the form (6) for some functions $A^{a}$ and $V$. Any admitted multiplier matrix $g_{a b}$, not a multiple of the identity and independent of the velocities, must be constant, must commute with the matrix of connection coefficients, must satisfy the conditions (9) with respect to the function $V$ and the curvature type condition (11) must hold with respect to the functions $A^{a}$. 
In the next section, we shall see that these conditions entail the existence of a linear change of coordinates which will partially decouple the given equations.

Functions $V$ which satisfy (9) have some remarkable properties. Since $g$ is constant, (9) can be written in the form

$$
\frac{\partial}{\partial x^{b}}\left(g_{a c} \frac{\partial V}{\partial x^{c}}\right)=\frac{\partial}{\partial x^{a}}\left(g_{b c} \frac{\partial V}{\partial x^{c}}\right),
$$

and hence

$$
g_{a c} \frac{\partial V}{\partial x^{c}}=\frac{\partial \xi}{\partial x^{a}} \quad \text { or equivalently } \quad \frac{\partial V}{\partial x^{a}}=g^{a c} \frac{\partial \xi}{\partial x^{c}},
$$

for some function $\xi$ on $\mathbb{R}^{n}$. The resulting integrability conditions to be satisfied by $V$ indicate that $\xi$ will itself be a solution of the equation (9). Alternatively, if we first multiply (9) by $g^{r a} g^{s b}$, we can write it in the form

$$
\frac{\partial}{\partial x^{r}}\left(g^{s b} \frac{\partial V}{\partial x^{b}}\right)=\frac{\partial}{\partial x^{s}}\left(g^{r a} \frac{\partial V}{\partial x^{a}}\right),
$$

from which a similar Bäcklund type transformation is obtained, by putting

$$
g^{s b} \frac{\partial V}{\partial x^{b}}=\frac{\partial \psi}{\partial x^{s}} \quad \text { or equivalently } \quad \frac{\partial V}{\partial x^{r}}=g_{r a} \frac{\partial \psi}{\partial x^{a}},
$$

for some function $\psi$. We thus obtain a double procedure to construct, in principle, new admissible potentials from known particular solutions.

Proposition 2. Let $\left(g_{a b}\right)$ be an arbitrary constant, non-singular, symmetric matrix, and assume $V_{0}(x)$ is a particular solution of the equation (9). Then, putting

$$
\frac{\partial V}{\partial x^{a}}=g^{a b} \frac{\partial V_{0}}{\partial x^{b}}, \quad \text { or } \quad \frac{\partial V}{\partial x^{a}}=g_{a b} \frac{\partial V_{0}}{\partial x^{b}},
$$

produces two sets of partial differential equations for $V$ which are guaranteed to be integrable and will produce other solutions of the same equation (9).

\section{Decoupling and a constructive characterization of the SODEs under consideration}

Given a second, constant multiplier matrix $g=\left(g_{a b}\right)$ arising from proposition 1, there then exists a constant orthogonal matrix $P$ which diagonalises it, $P^{T} g P=\operatorname{diag}\left(\lambda_{1} \ldots \lambda_{n}\right)$ say. The matrix $P$ can be regarded as the Jacobian of a linear coordinate transformation,

$$
x^{a}=P_{a b} y^{b}, \quad \text { with inverse } \quad y^{a}=P_{b a} x^{b} .
$$

Considering the Lagrangian $L_{0}$ which produces the given SODE with $\delta_{a b}$ as multiplier, it is clear that an arbitrary orthogonal coordinate transformation fully respects the structure of the system, i.e. in the new coordinates the system still has the unit matrix as 
multiplier and admits a second constant multiplier $g_{a b}$. Nevertheless, it is a worthwhile exercise (left to the reader) to double check that all concepts and conditions described in proposition 1 behave well under such a coordinate transformation. This may be obvious for $g$ and $\Phi$, for example, which are tensor fields, but less obvious for the matrix of connection coefficients, $\left(\Gamma_{b}^{a}\right)$, which certainly does not behave tensorially under arbitrary coordinate transformations. To be concrete, if $\bar{\Gamma}_{b}^{a}$ denotes the connection coefficients in new coordinates defined by (12), then

$$
\bar{\Gamma}_{b}^{a}=\frac{1}{2}\left(\frac{\partial \bar{A}^{a}}{\partial x^{b}}-\frac{\partial \bar{A}^{b}}{\partial x^{a}}\right), \quad \text { with } \quad \bar{A}^{a}=P_{b a} A^{b} .
$$

According to the elementary matrix considerations explained in the appendix, the orthogonal transformation which diagonalises $g$ will simultaneously transform $\Phi$ and $\Gamma_{b}^{a}$, which commute with $g$, into a corresponding block diagonal structure. Reversing the arguments, we are actually looking here at a property which fully characterises the systems under consideration, and can be expressed as follows.

Proposition 3. Assume we have a Lagrangian, $L_{0}$, for the given SODE, with a Euclidean kinetic energy term. Then the necessary and sufficient conditions for the existence of an alternative, constant multiplier $g$ are that $\Phi$ and the matrix of connection coefficients $\Gamma_{b}^{a}$ have common constant invariant subspaces, of which there are at least two.

Proof. The necessity of the conditions has been obtained above. Concerning the sufficiency, we reiterate that these conditions have an intrinsic meaning so long as we restrict ourselves to coordinate transformations which preserve the Euclidean structure, i.e. are of the form (12). Then, given these common invariant subspaces, it suffices to construct a corresponding block diagonal $g$ with a single eigenvalue for each of the blocks, making sure that at least two of these eigenvalues are distinct, so that $g$ does not become a multiple of the identity after diagonalisation.

We can do even better, by explaining how the block diagonal structure referred to above will actually lead to a decoupled system of differential equations. With $g$ diagonalised and $\Phi$ in block form, each of the blocks of $\Phi$ is spanned by a number of coordinate vector fields and the dimension of this substructure comes from the maximal degeneracy of eigenvalues of $g$. Thus we can define a number of disjoint distributions, $\mathcal{D}_{A}, A=1, \ldots, r$ say, where

$$
\mathcal{D}_{A}:=\operatorname{Sp}\left\{\frac{\partial}{\partial y^{A \alpha}}, \alpha=1, \ldots, \operatorname{dim} \mathcal{D}_{A}\right\}
$$

such that in a neighbourhood of an arbitrary point, we have $T \mathbb{R}^{n}=\oplus_{A=1}^{r} \mathcal{D}_{A}$. The $\mathcal{D}_{A}$ are basic distributions which are invariant under $\Phi$, by construction. Moreover, we have

$$
\nabla \frac{\partial}{\partial y^{A \alpha}}=\sum_{B, \beta} \bar{\Gamma}_{A \alpha}^{B \beta} \frac{\partial}{\partial y^{B \beta}}=\sum_{\beta} \bar{\Gamma}_{A \alpha}^{A \beta} \frac{\partial}{\partial y^{A \beta}},
$$

since $\bar{\Gamma}_{b}^{a}$ has the same block structure. Hence, we also have that $\nabla \mathcal{D}_{A} \subset \mathcal{D}_{A}$. 
It is known (see e.g. Proposition 1 in [14]) that the existence of a basic distribution, invariant under $\Phi$ and $\nabla$, is the necessary and sufficient condition for a SODE to be locally submersive in the sense defined in [8]. This means that in coordinates adapted to the distribution the second-order equations will display a feature of partial decoupling. But we have a much stronger situation here, namely a direct sum of a number of such distributions and the $y$-coordinates are already adapted to all of the $\mathcal{D}_{A}$. This means that the linear transformation to the $y$-coordinates will decouple the given SODE into a number of separate second-order systems of lower dimension.

Proposition 4. Assume that a given SODE $\Gamma$ satisfies the conditions of proposition 1 . Then the linear coordinate transformation which diagonalises the second constant multiplier $g_{a b}$ will decouple the system into a number of separate SODEs, one for each distinct eigenvalue of $g$.

This strong conclusion can be understood in terms of the conditions for decoupling established by Ferrario et al [5]. In that paper a SODE $\Gamma$ is assumed to admit two Lagrangians and a type $(1,1)$ tensor field $T$ on $T M$ is constructed out of the product of one of the multipliers with the inverse of the other. Decoupling follows if $T$ is diagonalisable, has even dimensional eigenspaces with constant degeneracy, has zero Nijenhuis torsion, commutes with the vertical endomorphism $S$ and is invariant under $\Gamma$. To see how our results fit into that theory, it suffices to define $g_{b}^{a}=g^{a c} \delta_{c b}$. This gives the (constant) components of a $(1,1)$ tensor on $M$, whose horizontal lift defines a tensor on $T M$, which is easily seen to satisfy all conditions of the theorem in [5].

Another interesting point is that, in agreement with the sufficiency statement in proposition 3 , the results of proposition 4 can actually be reversed and so provide a concrete procedure to construct SODEs which have both the unit matrix and another (constant) matrix as multiplier for the inverse problem. To this end, take any number of separate SODEs $\Gamma_{i}$ which are of the form of generalised electromagnetic forces, i.e. have right-hand sides of the form (6) and admit a corresponding standard Lagrangian $L_{i}$, with a scalar and vector potential and the Euclidean metric for the kinetic energy part. Multiply each $\Gamma_{i}$ with an arbitrary constant $\lambda_{i}$ creating a diagonal multiplier for the direct sum system $\Gamma=\oplus \Gamma_{i}$. Finally, apply an arbitrary linear, orthogonal coordinate transformation to this system. This will transform $\Gamma$ into a system which looks completely coupled and still has the identity matrix as multiplier; at the same time, the function $\sum \lambda_{i} L_{i}$ will be transformed into an alternative Lagrangian for $\Gamma$, corresponding to a second constant multiplier which has the chosen constants $\lambda_{i}$ as eigenvalues.

This is not the procedure we would apply if the starting point is a given SODE with right-hand sides of type (6) and we want to find for which scalar potentials $V$ and vector potentials $A^{a}$ there exist alternative multipliers. This is the way we will approach the characterization in the examples in the next section. 


\section{Examples}

Consider a system $\ddot{x}^{a}=F^{a}$, where the $F^{a}$ are of the form (6). A practical implementation of the results of proposition 1 works as follows: (i) first compute the matrix $\gamma$ with components $\gamma_{a b}=g_{a c} \Gamma_{b}^{c}$ and impose that $\gamma$ must be skew-symmetric; (ii) require that the curvature conditions (11) must hold; (iii) investigate the requirements (9) for admissible functions $V$. With a little luck, we will know the admissible vector potentials after the first two steps (or alternatively have fixed the freedom in the constant multiplier for given vector potentials), and can then address the remaining question about admissible potentials $V$ in the third step. The utility of propositions 2 and 3 will be illustrated as part of this process.

For a start, take $n=2$. The skew-symmetry of $\gamma$ requires that

$$
g_{12} \Gamma_{2}^{1}=0 \quad \text { and } \quad\left(g_{11}-g_{22}\right) \Gamma_{2}^{1}=0 .
$$

If $\Gamma_{2}^{1}$ were not zero, there would be no $g$ other than a multiple of the identity, in other words: given a scalar potential the addition of a magnetic (or gyroscopic) type force in dimension 2 immediately fixes the multiplier. So we must require that $\Gamma_{2}^{1}=0$ and the problem is reduced to the study of the condition

$$
g_{12}\left(\frac{\partial^{2} V}{\partial x_{2}^{2}}-\frac{\partial^{2} V}{\partial x_{1}^{2}}\right)=\left(g_{22}-g_{11}\right) \frac{\partial^{2} V}{\partial x_{1} \partial x_{2}} .
$$

By way of example, we proceed now to determine all admissible quadratic and cubic potentials. First, put

$$
V:=\frac{1}{2} k x_{1}^{2}+l x_{1} x_{2}+\frac{1}{2} m x_{2}^{2},
$$

Then the condition becomes

$$
g_{12}(m-k)=\left(g_{22}-g_{11}\right) l .
$$

If $l=0$ and $m=k$, then we are in the rather trivial situation where $\Phi$ is a multiple of the identity and every constant (non-singular) $g$ will be a multiplier (the given differential equations are in fact decoupled from the start). If only $l=0$ or only $m=k$, then $g_{12}$ must be zero in the first case, or $g_{11}=g_{22}$ in the second; in both cases, we have a 2-parameter family of multipliers still. The more interesting case is the generic situation that $l \neq 0$ and $m \neq k$. Then, if we put $l=(m-k) c$, the multiplier must have the form

$$
g=\left(\begin{array}{cc}
a & (b-a) c \\
(b-a) c & b
\end{array}\right)
$$

for some constants $a, b, c$ and the corresponding admissible potentials read

$$
V=\frac{1}{2} k x_{1}^{2}+(m-k) c x_{1} x_{2}+\frac{1}{2} m x_{2}^{2} .
$$


If we regard such a potential as given, for example, the 2-parameter family of corresponding Lagrangians we obtained reads,

$$
\begin{aligned}
L= & \frac{1}{2} a \dot{x}_{1}^{2}+\frac{1}{2} b \dot{x}_{2}^{2}+(b-a) c \dot{x}_{1} \dot{x}_{2} \\
& -\frac{1}{2}(b-a)(m-k) c^{2}\left(x_{1}^{2}+x_{2}^{2}\right)-\frac{1}{2} a k x_{1}^{2}-c(b m-a k) x_{1} x_{2}-\frac{1}{2} b m x_{2}^{2} .
\end{aligned}
$$

Take now, for example, the case that $a=1, b=2, c=1$, so that $l=m-k$. Computing the eigenvalues and eigenvectors of $g$, we find the matrix $P$ which, according to proposition 4 , should provide a linear coordinate transformation for decoupling the system. In fact, since an overall factor in such a transformation (12) does not matter for this respect, it is not necessary to normalise the eigenvectors. The transformation

$$
\begin{aligned}
& y_{1}:=2 x_{1}+(1+\sqrt{5}) x_{2}, \\
& y_{2}:=2 x_{1}+(1-\sqrt{5}) x_{2},
\end{aligned}
$$

does the job and the system decouples as

$$
\begin{aligned}
& \ddot{y}_{1}=-\left(k+\frac{1}{2}(1+\sqrt{5})(m-k)\right) y_{1}, \\
& \ddot{y}_{2}=-\left(k+\frac{1}{2}(1-\sqrt{5})(m-k)\right) y_{2} .
\end{aligned}
$$

For a test of proposition 2, still with the above choice of the parameters, let $V_{0}$ be a potential of the appropriate form and consider any of the following sets of equations for a new function $V$,

$$
\left\{\begin{array} { l } 
{ \frac { \partial V } { \partial x _ { 1 } } = \frac { \partial V _ { 0 } } { \partial x _ { 1 } } + \frac { \partial V _ { 0 } } { \partial x _ { 2 } } , } \\
{ \frac { \partial V } { \partial x _ { 2 } } = \frac { \partial V _ { 0 } } { \partial x _ { 1 } } + 2 \frac { \partial V _ { 0 } } { \partial x _ { 2 } } , }
\end{array} \quad \text { or } \quad \left\{\begin{array}{l}
\frac{\partial V}{\partial x_{1}}=2 \frac{\partial V_{0}}{\partial x_{1}}-\frac{\partial V_{0}}{\partial x_{2}} \\
\frac{\partial V}{\partial x_{2}}=-\frac{\partial V_{0}}{\partial x_{1}}+\frac{\partial V_{0}}{\partial x_{2}}
\end{array}\right.\right.
$$

These equations are easily seen to be integrable and produce potentials belonging to the same family.

Next we turn to cubic potentials on $\mathbb{R}^{2}$ and set

$$
V:=\frac{1}{3} k x_{1}^{3}+l x_{1}^{2} x_{2}+m x_{1} x_{2}^{2}+\frac{1}{3} n x_{2}^{3} .
$$

The requirement that $\gamma$ be skew-symmetric now produces two conditions, namely

$$
\begin{aligned}
g_{12}(m-k) & =\left(g_{22}-g_{11}\right) l, \\
g_{12}(n-l) & =\left(g_{22}-g_{11}\right) m .
\end{aligned}
$$

We shall not go into a detailed discussion of subcases this time. So, in a generic situation, the two conditions above are compatible only if $m(m-k)=l(n-l)$. For comparison, let us put $l=(m-k) c$ again, so that $m=(n-l) c$. The multiplier $g$ then takes exactly the same form as in the quadratic case, and the corresponding admissible potentials read

$$
V=\frac{1}{3} k x_{1}^{3}+\frac{c(n c-k)}{1+c^{2}} x_{1}^{2} x_{2}+\frac{c(n+c k)}{1+c^{2}} x_{1} x_{2}^{2}+\frac{1}{3} n x_{2}^{3} .
$$


To be more concrete, taking $a=1, b=2, c=1$ as before, we leave it to the reader to verify that the Bäcklund transformations of proposition 2 perform their task again, and that the same coordinate transformation to new variables $\left(y_{1}, y_{2}\right)$ will decouple the system.

We now turn to systems with three degrees of freedom, where we can expect vector potentials to enter the discussion. Taking the symmetry of $g$ and skew-symmetry of $\Gamma_{b}^{a}$ into account, the skew-symmetry of $\gamma$ first of all requires that

$$
g_{12} \Gamma_{2}^{1}+g_{13} \Gamma_{3}^{1}=0, \quad g_{12} \Gamma_{2}^{1}-g_{23} \Gamma_{3}^{2}=0, \quad g_{13} \Gamma_{3}^{1}+g_{23} \Gamma_{3}^{2}=0 .
$$

Except for special cases where one or more of the $\Gamma_{b}^{a}$ vanish (and which we will not discuss), these relations indicate firstly that the ratio of any two $\Gamma_{b}^{a}$ must be constant. So, assuming that $\Gamma_{2}^{1} \neq 0$, we can put

$$
\Gamma_{3}^{1}=a \Gamma_{2}^{1}, \quad \Gamma_{3}^{2}=b \Gamma_{2}^{1}, \quad a, b \text { constant. }
$$

It follows that $g_{12}=-a g_{13}=b g_{23}$. The off diagonal elements of $\gamma$ impose three more conditions, only two of which are independent, say

$$
\left(g_{11}-g_{22}\right) \Gamma_{2}^{1}=g_{13} \Gamma_{3}^{2}+g_{23} \Gamma_{3}^{1}, \quad\left(g_{11}-g_{33}\right) \Gamma_{3}^{1}=g_{23} \Gamma_{2}^{1}-g_{12} \Gamma_{3}^{2} .
$$

Since $g$ is only determined up to an overall factor, we can set without loss of generality $g_{13}=-b$, which implies that $g_{23}=a$ and $g_{12}=a b$. If we further rename $g_{11}$ as the constant $c$, we finally obtain the following structure of the multiplier $g$ :

$$
g=\left(\begin{array}{ccc}
c & a b & -b \\
a b & c+b^{2}-a^{2} & a \\
-b & a & c+b^{2}-1
\end{array}\right)
$$

In dimension three, the curvature type condition contains one requirement, which can be seen to reduce to

$$
\frac{\partial \Gamma_{2}^{1}}{\partial x_{3}}-a \frac{\partial \Gamma_{2}^{1}}{\partial x_{2}}+b \frac{\partial \Gamma_{2}^{1}}{\partial x_{1}}=0 .
$$

The general solution of this equation is found to be an arbitrary function of two variables, namely

$$
u:=x_{1}-b x_{3}, \quad v:=x_{2}+a x_{3} .
$$

This means that admissible vector potentials must satisfy

$$
\begin{aligned}
& \frac{1}{2}\left(\frac{\partial A_{1}}{\partial x_{2}}-\frac{\partial A_{2}}{\partial x_{1}}\right)=f(u, v), \\
& \frac{1}{2}\left(\frac{\partial A_{1}}{\partial x_{3}}-\frac{\partial A_{3}}{\partial x_{1}}\right)=a f(u, v), \\
& \frac{1}{2}\left(\frac{\partial A_{2}}{\partial x_{3}}-\frac{\partial A_{3}}{\partial x_{2}}\right)=b f(u, v),
\end{aligned}
$$


for some arbitrary $f$. Using the homotopy formula from the standard proof of the Poincaré lemma, we can actually give an explicit expression for the components $A_{a}$, which reads

$$
\begin{aligned}
& A_{1}=2 \int_{0}^{1} t v f(t u, t v) d t, \\
& A_{2}=-2 \int_{0}^{1} t u f(t u, t v) d t, \\
& A_{3}=-2 \int_{0}^{1} t(a u+b v) f(t u, t v) d t .
\end{aligned}
$$

Now we are left with the conditions for admissible scalar potentials. There are three of them, one of which reads

$$
\left(g_{11}-g_{22}\right) \frac{\partial^{2} V}{\partial x_{1} \partial x_{2}}+g_{12}\left(\frac{\partial^{2} V}{\partial x_{2}^{2}}-\frac{\partial^{2} V}{\partial x_{1}^{2}}\right)=g_{23} \frac{\partial^{2} V}{\partial x_{1} \partial x_{3}}-g_{13} \frac{\partial^{2} V}{\partial x_{2} \partial x_{3}} .
$$

The other two are similar in structure. Using the information we already gathered, two of these equations can be written in the form,

$$
\begin{aligned}
\left(1-b^{2}\right) \frac{\partial^{2} V}{\partial x_{1} \partial x_{3}}-b\left(\frac{\partial^{2} V}{\partial x_{3}^{2}}-\frac{\partial^{2} V}{\partial x_{1}^{2}}\right) & =a \frac{\partial^{2} V}{\partial x_{1} \partial x_{2}}-a b \frac{\partial^{2} V}{\partial x_{2} \partial x_{3}}, \\
\left(1-a^{2}\right) \frac{\partial^{2} V}{\partial x_{2} \partial x_{3}}+a\left(\frac{\partial^{2} V}{\partial x_{3}^{2}}-\frac{\partial^{2} V}{\partial x_{2}^{2}}\right) & =-b \frac{\partial^{2} V}{\partial x_{1} \partial x_{2}}-a b \frac{\partial^{2} V}{\partial x_{1} \partial x_{3}} .
\end{aligned}
$$

The third one, actually the one we displayed first, is just a linear combination of these two. Once again and quite remarkably, we can obtain the general solution of these equations. A sketch of the way one can proceed goes as follows. Inspired by the general solution for the vector potential, we perform a linear coordinate transformation from the variables $\left(x_{1}, x_{2}, x_{3}\right)$ to the variables $\left(u, v, x_{3}\right)$. In those variables, the above two requirements reduce to

$$
\begin{aligned}
& \left(1+b^{2}\right) \frac{\partial V}{\partial u}-a b \frac{\partial V}{\partial v}-b \frac{\partial V}{\partial x_{3}}=\zeta(u, v), \\
& \left(1+a^{2}\right) \frac{\partial V}{\partial v}-a b \frac{\partial V}{\partial u}+a \frac{\partial V}{\partial x_{3}}=\eta(u, v),
\end{aligned}
$$

where $\zeta$ and $\eta$ are as yet arbitrary functions of the indicated variables. Eliminating the derivatives with respect to $x_{3}$ in these equations, we find that $a(\partial V / \partial u)+b(\partial V / \partial v)$ must be a function of $u$ and $v$ only. This is an equation one can integrate and the immediate result is that $V$ must be a sum of a function of $u$ and $v$ only, plus a function $W$ of two variables also, namely $x_{3}$ and $w:=a v-b u$. It is easy to verify that an arbitrary function of $u$ and $v$ satisfies the requirements for the potential $V$. Since the equations are linear, it suffices to see whether the same conclusion holds for $W\left(x_{3}, w\right)$. If we insert such a function into the last two displayed equations for $V$, they both reduce to an equation which requires that

$$
\left(1+a^{2}+b^{2}\right) \frac{\partial W}{\partial w}+\frac{\partial W}{\partial x_{3}}
$$


must be a function of $w$ only. This in turn implies that $W$ is the sum of a function of $w$ only, plus another function of only one variable, namely $z:=\left(1+a^{2}+b^{2}\right) x_{3}-w$, which in terms of the original variables reads

$$
z=x_{3}+b x_{1}-a x_{2} .
$$

The first part of $W$ can be absorbed into the arbitrary function of $u, v$ in $V$, so that the final answer for the general expression for admissible potentials reads:

$$
V=U(u, v)+Z(z),
$$

where $U$ and $Z$ are arbitrary functions of the indicated arguments.

Recapitulating, we can state that for $n=3$, in the generic case that none of the $\Gamma_{b}^{a}$ with $a \neq b$ are zero, the most general systems satisfying the conditions of proposition 1 , are such that a multiplier $g$, different from the identity, will be of the form (13), the corresponding admissible vector potential is given by (15), and the scalar potential by (17), where $u, v, z$ are defined by (14) and (16), and $f, U$ and $Z$ are arbitrary functions of the indicated variables.

The multiplier $g$ has two distinct eigenvalues, namely $c-a^{2}-1$ and the double eigenvalue $c+b^{2}$. As before, one does not necessarily have to use strictly the orthogonal matrix $P$ which diagonalises $g$ to find the transformation for decoupling the system. Not surprisingly, the transformation to the variables $u, v, z$ will decouple the system into a coupled system of two equations for $u$ and $v$, and a single equation for $z$ :

$$
\begin{aligned}
\ddot{u} & =-2\left(a b \dot{u}+\left(1+b^{2}\right) \dot{v}\right) f(u, v)-\left(1+b^{2}\right) \frac{\partial U}{\partial u}+a b \frac{\partial U}{\partial v}, \\
\ddot{v} & =2\left(\left(1+a^{2}\right) \dot{u}+a b \dot{v}\right) f(u, v)+a b \frac{\partial U}{\partial u}-\left(1+a^{2}\right) \frac{\partial U}{\partial v}, \\
\ddot{z} & =-\left(1+a^{2}+b^{2}\right) Z^{\prime}(z) .
\end{aligned}
$$

Here is an explicit example of alternative Lagrangians (in fact another 2-parameter family of examples). Consider the following given SODE, where $a$ and $b$ are arbitrary constants:

$$
\begin{aligned}
& \ddot{x}_{1}=-2 f(x)\left(\dot{x}_{2}+a \dot{x}_{3}\right)-\frac{2}{3}\left(x_{1}-b x_{3}\right)^{2}-b\left(x_{3}+b x_{1}-a x_{2}\right), \\
& \ddot{x}_{2}=2 f(x)\left(\dot{x}_{1}-b \dot{x}_{3}\right)+a\left(x_{3}+b x_{1}-a x_{2}\right), \\
& \ddot{x}_{3}=2 a f(x) \dot{x}_{1}+2 b f(x) \dot{x}_{2}+\frac{2}{3} b\left(x_{1}-b x_{3}\right)^{2}-\left(x_{3}+b x_{1}-a x_{2}\right),
\end{aligned}
$$

with

$$
f(x):=x_{1} x_{2}+a x_{1} x_{3}-b x_{2} x_{3}-a b x_{3}^{2} .
$$

One easily verifies that this particular family of soDEs comes from the general results established above, where we have chosen the arbitrary functions $f(u, v), U(u, v), Z(z)$ to be: $f=u v, U=\frac{1}{3} u^{3}, Z=\frac{1}{2} z^{2}$. The Lagrangian with standard kinetic energy for this SODE, written in a form such as (5), reads:

$$
L_{0}:=\frac{1}{2} \sum \dot{x}_{i}^{2}+\frac{1}{2}\left(u v^{2} \dot{x}_{1}-u^{2} v \dot{x}_{2}-\left(a u^{2} v+b u v^{2}\right) \dot{x}_{3}\right)-\frac{1}{3} u^{3}-\frac{1}{2} z^{2} .
$$


Following our generic results, alternative Lagrangians for this system are obtained from multipliers of the form (2). Since such $g$ are determined up to a scalar factor anyway, we simply put $c=1$. It then follows after some calculations that the corresponding alternative Lagrangians, again specifically written in the representation (5) which refers to the original coordinates, are given by

$$
\begin{aligned}
L= & \frac{1}{2} \dot{x}_{1}^{2}+\frac{1}{2}\left(b^{2}-a^{2}+1\right) \dot{x}_{2}^{2}+\frac{1}{2} b \dot{x}_{3}^{2}+a b \dot{x}_{1} \dot{x}_{2}-b \dot{x}_{1} \dot{x}_{3}+a \dot{x}_{2} \dot{x}_{3} \\
& +\frac{1}{2}\left(1+b^{2}\right)\left(u v^{2} \dot{x}_{1}-u^{2} v \dot{x}_{2}-\left(a u^{2} v+b u v^{2}\right) \dot{x}_{3}\right)-\frac{1}{3}\left(1+b^{2}\right) u^{3}+\frac{1}{2} a^{2} z^{2} .
\end{aligned}
$$

\section{The Coulomb problem}

In the physical realms of $n=2$ and $n=3$, the practical implementation of the conditions of proposition 1 can be seen from different angles. For example, we could start from a given, fixed potential $V$. Then, the conditions (9) for admissible potentials become algebraic relations which will restrict the existence of alternative multipliers. And if such an alternative multiplier can be found, it is clear that turning on an extra magnetic field type force, will further severely restrict the possibility of having such $g$ (or simply make it impossible) through the extra algebraic restrictions that $\gamma_{a b}=g_{a c} \Gamma_{b}^{c}$ must be skew-symmetric (as we pointed out in the $n=2$ case in the last section). Equivalently, the matrices with components $\Phi_{b}^{a}$ and $\Gamma_{b}^{a}$ will now fail the conditions of proposition 3 . In this section, as an aside, we exemplify this effect of adding a magnetic field type force to a scalar potential in a more general setting in which the multiplier may depend on the velocities.

Consider the classical problem of the motion of a particle under the influence of a Coulomb potential. Putting the mass equal to 1 for simplicity, the equations of motion are of the form $\ddot{\mathbf{r}}=-\left(k / r^{3}\right) \mathbf{r}$. Clearly, the system has the unit matrix as multiplier, but it is a simple exercise for the reader to show that no other constant multiplier exists. Yet, it is known that there are alternative Lagrangians (see $[7,4]$ ) when velocity dependence is allowed in the multiplier. For example,

$$
L_{\gamma}:=L_{0}+\gamma \frac{J}{r^{2}}
$$

where $\gamma$ is a real constant and $J$ is the magnitude of the conserved angular momentum. As discussed in [7] the quantum mechanics arising from these alternative Lagrangians are different from the standard hydrogen atom and some sort of selection rule appears to be required. We will show here that adding a constant magnetic field to the system has the effect of removing the ambiguity in the choice of a Lagrangian. The quantum mechanics is then unambiguously the hydrogen atom experiencing the Zeeman effect. At a quantum mechanical level one can take the zero magnetic field limit and recover the standard hydrogen atom. [The reader is referred to [12] and references therein to see a discussion of alternative Lagrangians for a particle in a magnetic field alone.] 
As usual we choose the $z$-axis along the given constant magnetic field vector. The modified system still has the unit matrix as multiplier in the cartesian coordinates, but it will be more convenient here to work in cylindrical coordinates $\rho, \theta, z$. In those coordinates, the equations of motion read

$$
\begin{aligned}
\ddot{\rho} & =\rho \dot{\theta}^{2}+B \rho \dot{\theta}-k \frac{\rho}{r^{3}}, \\
\ddot{\theta} & =-2 \frac{\dot{\rho} \dot{\theta}}{\rho}-B \frac{\dot{\rho}}{\rho} \\
\ddot{z} & =-k \frac{z}{r^{3}},
\end{aligned}
$$

where $r^{2}=\rho^{2}+z^{2}$. The standard multiplier is now $\operatorname{diag}\left(1, \rho^{2}, 1\right)$. We apply the full inverse problem machinery now in search of a possible second multiplier (allowed to depend on coordinates and velocities).

The algebraic conditions $g_{a b} \Phi_{c}^{b}=g_{c b} \Phi_{a}^{b}$ produce the following restrictions on the elements of the symmetric matrix $g$ :

$$
\begin{aligned}
& \rho g_{12}+z g_{23}=0, \\
& 12 k\left(\left(g_{11}-g_{33}\right) \rho z+g_{13}\left(z^{2}-\rho^{2}\right)\right)+B^{2} r^{5} g_{13}=0, \\
& 12 k\left(g_{12} \rho z+g_{23} z^{2}\right)+B^{2} r^{5} g_{23}=0 .
\end{aligned}
$$

From the first and third of these, it follows that we must have

$$
g_{23}=g_{12}=0 .
$$

With this information, the differential conditions $\nabla g=0$ (the second of the conditions (3) imply first of all that also $g_{13}$ must be zero, and hence from the second condition above that $g_{11}=g_{33}$, and further indicate that $g_{22}=\rho^{2} g_{11}$, that $g_{11}$ (and thus $g_{33}$ ) must be first integrals, and that $g_{22}$ must satisfy the condition

$$
\rho \Gamma\left(g_{22}\right)-2 \dot{\rho} g_{22}=0 .
$$

Finally, the last of the conditions (3) imply that we must have

$$
\frac{\partial g_{22}}{\partial \dot{\rho}}=\frac{\partial g_{12}}{\partial \dot{\theta}}=0 \quad \text { in view of } g_{12}=0,
$$

and likewise that

$$
\frac{\partial g_{11}}{\partial \dot{\theta}}=\frac{\partial g_{12}}{\partial \dot{\rho}}=0 \quad \text { and } \quad \frac{\partial g_{22}}{\partial \dot{z}}=\frac{\partial g_{23}}{\partial \dot{\theta}}=0 .
$$

It then further follows from $g_{22}=\rho^{2} g_{11}$ that actually all derivatives of $g_{11}$ and $g_{22}$ with respect to velocity coordinates must vanish. But a first integral which does not depend on velocities necessarily is just a constant, so the conclusion is that the only multipliers we can obtain are simply constant multiples of the standard one we knew from the start. 


\section{Appendix: Some elementary matrix considerations}

It is well known that commuting symmetric matrices can be diagonalised simultaneously. Let us analyse some aspects of the way this works. Suppose $A^{T}=A, B^{T}=B$ and $A B=$ $B A$, so that also $(A B)^{T}=A B$. If $P$ denotes an orthogonal matrix which diagonalises $A$, say $P^{T} A P=\operatorname{diag}\left(\lambda_{1} \ldots \lambda_{n}\right)$, and put $B^{\prime}=P^{T} B P$. Then,

$$
P^{T} A B P=\operatorname{diag}\left(\lambda_{1} \ldots \lambda_{n}\right) B^{\prime}=P^{T} B A P=B^{\prime} \operatorname{diag}\left(\lambda_{1} \ldots \lambda_{n}\right),
$$

from which it follows that

$$
\lambda_{i} b_{i j}^{\prime}=b_{i j}^{\prime} \lambda_{j} \quad(\text { no sum! }) \quad \forall i, j,
$$

so that $b_{i j}^{\prime}=0$ when $\lambda_{i} \neq \lambda_{j}$. This means that $B^{\prime}$ will have a block diagonal structure, the dimension of each block corresponding to the multiplicity of an eigenvalue $\lambda_{i}$ of $A$. Looking at each block separately, there is a further orthogonal transformation which will diagonalise that block without affecting $A$, because $A$ is a multiple of the identity with respect to this substructure. This way the simultaneous diagonalisation can be achieved.

We are interested also in the case that one of the matrices is skew-symmetric. So suppose $A^{T}=A, B^{T}=-B$ and $A B=B A$. Then $(A B)^{T}=-A B$, and if $P$ is as before, the first step of the above procedure remains unchanged and leads to the conclusion that $B^{\prime}$, which is skew-symmetric also, has a block diagonal structure. This time, further orthogonal transformations which will not affect $A$ can bring each of the blocks of $B^{\prime}$ in 'diagonal form' in the sense of the real Jordan form. Again, this way, $A$ and $B$ have been simultaneously diagonalised in the sense just described.

\section{Acknowledgements}

This work results from mutual research visits of the authors to each others institutions. In addition W.S. is grateful for support and hospitality at the Australian Mathematical Sciences Institute in May/June 2010 and further acknowledges support from the Research Foundation - Flanders (FWO). G.P. thanks Alex Melitsis, Olga Krupková and Demeter Krupka for their input in discussions.

\section{References}

[1] J. E. Aldridge, G. E. Prince, W. Sarlet and G. Thompson, An EDS approach to the inverse problem in the calculus of variations, J. Math. Phys. 47 (2006) 103508.

[2] I. Anderson and G. Thompson, The inverse problem of the calculus of variations for ordinary differential equations, Mem. Amer. Math. Soc. 473 (1992).

[3] I. Bucataru and M.F. Dahl, Semi-basic 1-forms and Helmholtz conditions for the inverse problem of the calculus of variations, J. Geom. Mech. 1 (2009) 159-180. 
[4] M. Crampin and G.E. Prince, Generalising gauge variance for spherically symmetric potentials, J. Phys. A: Math. Gen. 18 (1985) 2167-2175.

[5] C. Ferrario, G. Lo Vecchio, G. Marmo, G. Morandi and C. Rubano, A separability theorem for dynamical systems admitting alternative Lagrangian descriptions, $J$. Phys. A: Math. Gen. 20 (1987) 3225-3236.

[6] J. Grifone and Z. Muzsnay, On the inverse problem of the variational calculus: existence of Lagrangians associated with a spray in the isotropic case, Ann. Inst. Fourier 49 (1999) 1387-1421.

[7] M. Henneaux and L.C. Shepley, Lagrangians for sperically symmetric potentials, $J$. Math. Phys.23 (1982) 2101-2107.

[8] M. Kossowski and G. Thompson, Submersive second-order differential equations, Math. Proc. Camb. Phil. Soc. 110 (1991) 207-224.

[9] O. Krupková and G. E. Prince, Second order ordinary differential equations in jet bundles and the inverse problem of the calculus of variations, in Handbook of Global Analysis D. Krupka and D. Saunders eds. (Elsevier 2008) 837-904.

[10] E. Martínez, J. F. Cariñena and W. Sarlet, Derivations of differential forms along the tangent bundle projection II, Differential Geom. Appl. 3 (1993) 1-29.

[11] T. Mestdag, W. Sarlet and M. Crampin, The inverse problem for Lagrangian systems with certain non-conservative forces, (2010) arXiv:1004.0674.

[12] G. Morandi, C. Ferrario, G. Lo Vecchio, G. Marmo and, C. Rubano, The inverse problem in the calculus of variations and the geometry of the tangent bundle, Physics Reports 188 Issues 3-4, (1990) 147-284.

[13] W. Sarlet, The Helmholtz conditions revisited. A new approach to the inverse problem of Lagrangian dynamics, J. Phys. A: Math. Gen. 15 (1982) 1503-1517.

[14] W. Sarlet and W. Vanbiervliet, Geometric characterization of driven cofactor systems, J. Phys. A: Math. Theor. 41 (2008) 042001 (10pp). 